\title{
PROPRIEDADES QUÍMICAS DE UM NEOSSOLO QUARTZARÊNICO, MARANHÃO, BRASIL
}

Gonçalo Mendes da Conceição ; Ana Cláudia Ruggieri²; Wilton Ladeira da Silva

${ }^{1}$ Professor Doutor do Centro de Estudos Superiores de Caxias/CESC, Universidade

Estadual do Maranhão/UEMA/Núcleo de Pesquisa dos Cerrados

Maranhenses/RBCEM, Laboratório de Biologia Vegetal/LABIVE

${ }^{2}$ Professora Doutora da Faculdade de Ciências Agrárias e

Veterinarias/UNESP/Jaboticabal/Departamento de Zootecnia

${ }^{3}$ Doutorando da Faculdade de Ciências Agrárias e

Veterinarias/UNESP/Jaboticabal/Departamento de Zootecnia

Recebido em: 03/01/2014 - Aprovado em: 04/04/2014 - Publicado em: 12/04/2014

\section{RESUMO}

A pesquisa foi realizada em uma área de cerrado, localizada no município de Caxias, pertencente á região Leste Maranhense. O município apresenta fitofisionomias que compreendem desde o campo cerrado até cerradão. Na área de estudo foram instaladas cinco transectos de $100 \mathrm{~m}$ cada, com interdistanciamento de $50 \mathrm{~m}$. Em cada transecção foram coletadas quatro amostras de solo, na profundidade de 0-20 $\mathrm{cm}$, nas quais foram determinadas a textura e características químicas. Os teores de argila variaram entre 3 e $5 \%$. O pH do solo variou de 4,63 a 4,82. Os teores de Cálcio e Magnésio foram baixos, variando de 0,22 a $0,40 \mathrm{cmo}_{\mathrm{c}} / \mathrm{dm}^{-3}$ no Cálcio, enquanto que o Magnésio de 0,25 a 0,42 $\mathrm{cmo}_{\mathrm{c}} / \mathrm{dm}^{-3}$. Os valores de Potássio não se diferiram, apresentando média de $0,02 \mathrm{cmo}_{\mathrm{c}} / \mathrm{dm}^{-3}$, enquanto o Fósforo variou de 2 a $9 \mathrm{mg} / \mathrm{dm}^{-3}$. Os teores de Matéria Orgânica variaram entre 9,33 e 23,03 g/ $\mathrm{kg}^{-1}$, valores estes elevados para ambientes tropicais.

PALAVRAS-CHAVE: nutrientes do solo, matéria orgânica, pastagem nativa, textura do solo.

\section{SOIL CHEMICAL PROPERTIES IN A TYPIC QUARTZIPISAMMENT, MARANHÃO STATE, BRAZIL}

\begin{abstract}
The research was conducted in an area of cerrado, located in the city of Caxias, belonging to the region east of Maranhão. The municipality has vegetation types that range from the field until cerrado savanna. In the study area five transects of $100 \mathrm{~m}$ each were installed with. In each transect four soil samples were collected at a depth of $0-20 \mathrm{~cm}$, which were determined in the texture and chemical characteristics. Clay contents ranged between 3 and $5 \%$. Soil pH ranged from 4.63 to 4.82 . The calcium and magnesium concentrations were low, ranging from 0.22 to $0.40 \mathrm{mg} / \mathrm{dm}^{3}$, Magnesium while $0.25-0.42 \mathrm{cmo}_{d} / \mathrm{dm}^{3}$. Potassium values do not differ and averaged $0.02 \mathrm{cmo}_{\mathrm{C}} / \mathrm{dm}^{3}$, while the Phosphorus ranged $2-9 \mathrm{mg} / \mathrm{dm}^{3}$. The content of organic matter ranged between 9.33 and $23.03 \mathrm{~g} / \mathrm{kg}^{-1}$, these high values for tropical
\end{abstract}


environments. It is inferred that the soil studied, the levels of soil organic matter are higher than typical for this class of soil.

KEYWORDS: Organic matter, soil texture, nutrients soil, native pasture

\section{INTRODUÇÃO}

A vegetação do Cerrado é composta por um mosaico (fitofisionomias) representado por formações florestais, savânicas e campestres (RIBEIRO \& WALTER, 2008). Dentre estas, a fitofisionomia do cerrado sensu stricto, de acordo com RIBEIRO \& VALTER (1998), caracteriza-se por apresentar dois estratos, um arbóreo e outro herbáceo bem definido, com árvores baixas, inclinadas, tortuosas, com ramificações irregulares e retorcidas, distribuídas aleatoriamente sobre o solo em diferentes densidades. Esta fisionomia ocupa aproximadamente $70 \%$ do bioma Cerrado (ASSUNÇÃO \& FELFILI, 2004), sendo, portanto, merecedora de preocupação, uma vez que a destruição do cerrado sensu lato acompanha o ritmo de supressão de sua principal paisagem que é o cerrado sensu stricto, conforme salientou NETTESHEIM et al. (2010).

O solo é um recurso que suporta toda a comunidade vegetal, sem a qual os seres vivos não poderiam existir. Nessa cobertura, incluem-se não só as culturas, como também todos os tipos de árvores, gramíneas e herbáceas que podem ser utilizadas pelo homem (BERTONI \& LOMBARDI NETO, 1985). É um ambiente natural, com corpos tridimensionais usados para diversas finalidades, sendo a mais importante a produção de alimentos e fibras para o gênero humano e animais domésticos (BRADY, 1989). Já EMBRAPA (2006) define solo como sendo uma coleção de corpos naturais, constituídos por partes sólidas, liquidas e gasosas, tridimensionais, dinâmicos, formados por materiais minerais e orgânicos que ocupam a maior parte do manto superficial das extensões continentais, contém matéria viva e podem ser vegetados na natureza onde ocorrem e, eventualmente, terem sido modificados por interferências antrópicas.

REATTO \& MARTINS (2005) definiram classicamente os solos e asseveram que estes são resultados de cinco variáveis interdependentes, denominadas fatores de formação do solo que são: clima, organismos, material de origem, relevo e tempo, que agem sobre as rochas, transformando-as em solos e sedimentos.

No Brasil os estudos sobre solos tiveram inicio, em caráter sistemático, no final da década de 50 , sendo seu maior desenvolvimento somente a partir dos anos 70 (ADÁMOLI et al., 1985).

Os solos do Cerrado refletem um equilíbrio frágil entre o relevo, o clima e vegetação. Em condições naturais, ocorre no Cerrado a erosão dos solos pelas águas de escoamento superficial e subsuperficial. As modalidades do escoamento superficial são determinadas pela distribuição das precipitações, pela natureza dos solos e da cobertura vegetal (PINTO \& BARROS, 1996).

Os solos do Cerrado são bastante ácidos, com pH variando de 4 a 5,5. Sendo que esta acidez é consequência, em boa parte aos altos níveis de $\mathrm{Al}^{3+}$, tornando-os aluminotóxicos para a maioria das culturas agrícolas. Também níveis elevados de íons de Fe e Mn, contribuem para a toxidez. Baixa CTC, baixa soma de bases e alta saturação por $\mathrm{Al}^{3+}$, caracterizam estes solos como profundamente distróficos, sendo, portanto, impróprios para a agricultura (COUTINHO, 2002). 


\section{MATERIAL E MÉTODOS}

A pesquisa foi realizada em uma área de Cerrado, localizada no município de Caxias/MA, nas coordenadas $04^{\circ} 53^{\prime} 30^{\prime \prime}$ e e $43^{\circ} 24^{\prime} 53^{\prime \prime}$ W, pertencente á região Leste Maranhense. Este município possui $5.224,02 \mathrm{~km}^{2}$ de área de cerrado (BRASIL, 2009), com fitofisionomias que compreendem desde o campo cerrado até o cerradão (Figura 1).

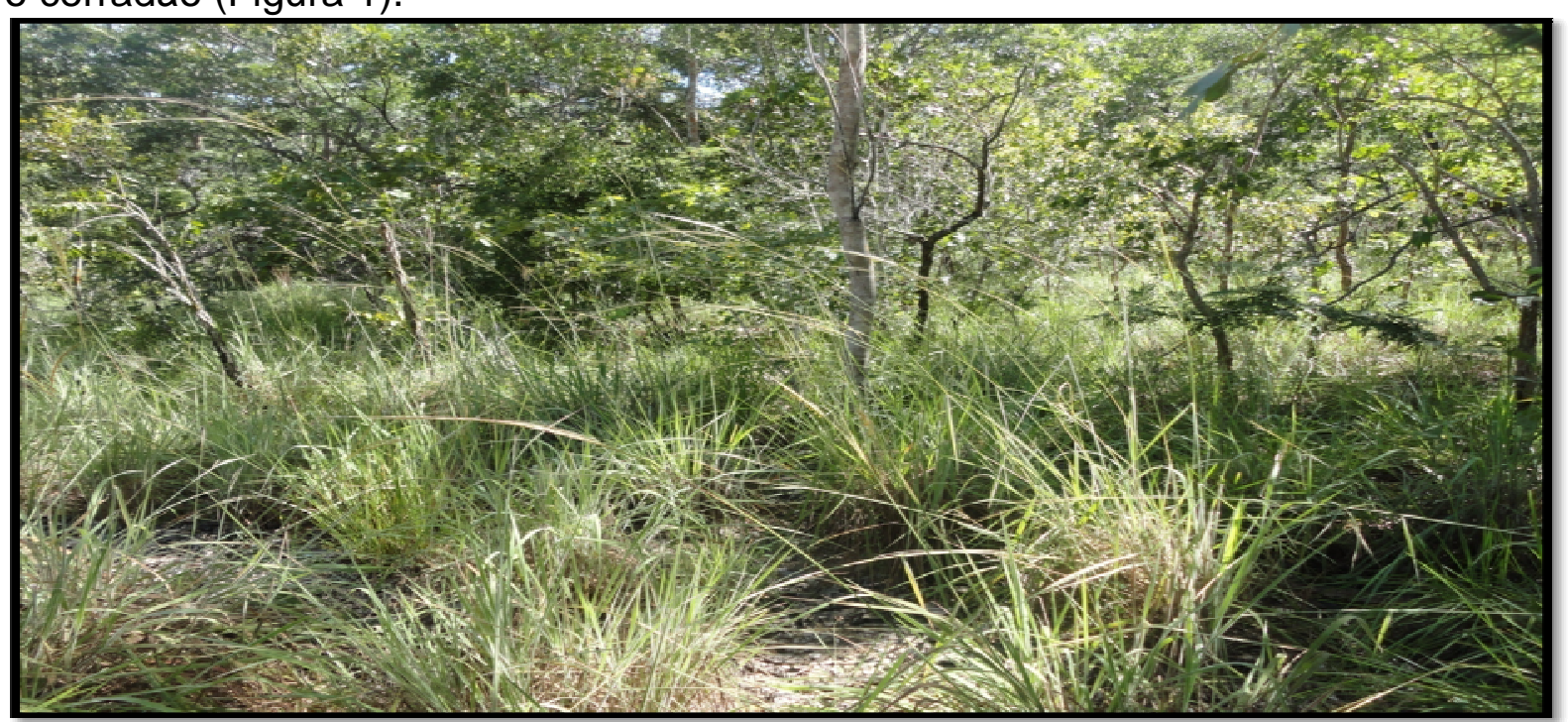

FIGURA 1. Aspecto fisionônomico do Cerrado pesquisado, apresentando dois estratos distintos: um herbáceo constituído principalmente por gramíneas e outro arbustivo-arbóreo.

O clima do município é classificado como Subúmido Seco, com temperatura máxima anual variando de $32,60^{\circ} \mathrm{C}$ (junho) e $37,50^{\circ} \mathrm{C}$ (outubro), com médias de $25,10^{\circ} \mathrm{C}$ (julho) e $28,70^{\circ} \mathrm{C}$ (outubro) e temperatura mínima de $16,70^{\circ} \mathrm{C}$ (outubro) e $17,20^{\circ} \mathrm{C}$ (setembro), conforme Figura 2 (CONCEIÇÃO et al., 2013).

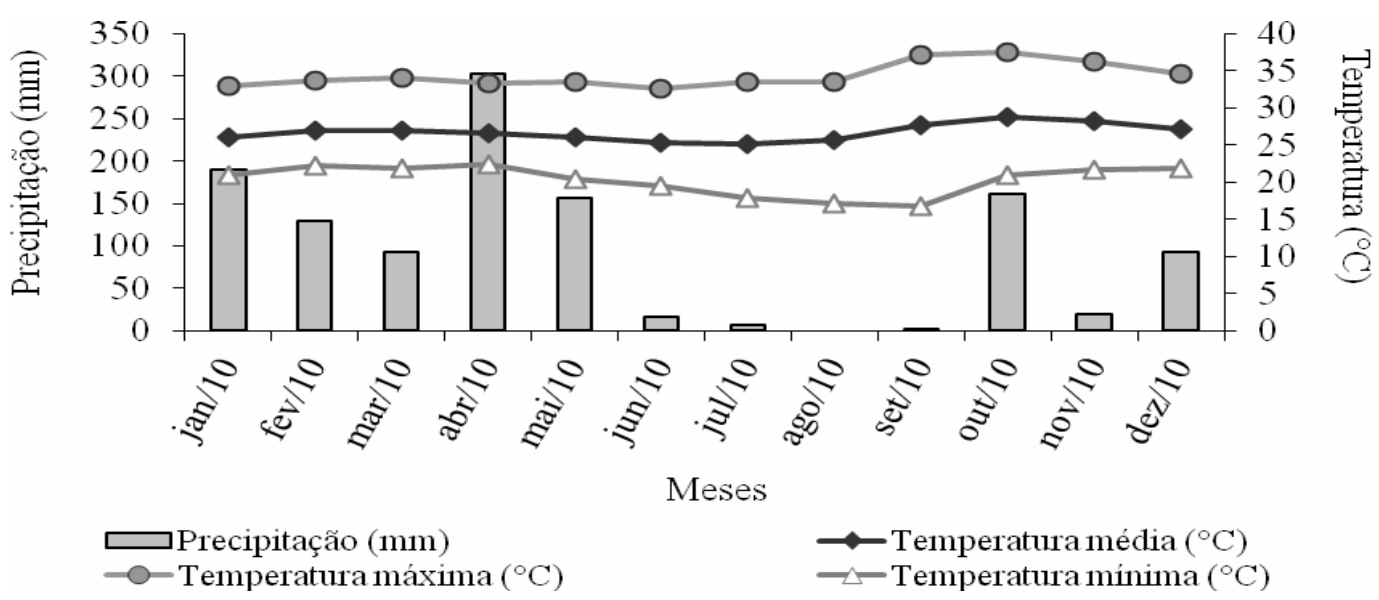

FIGURA 2. Valores médios de precipitação pluviométrica e temperatura máxima, média e mínima no ano de 2010, para o Município de Caxias/Maranhão (CONCEIÇÃO et al., 2013).

A pesquisa foi realizada na Propriedade Cupins, cortada pela BR 316, que liga o município de Caxias a Timon, ambos no Maranhão. A área de estudo 
caracteriza-se por apresentar uma fitofisionomia de cerrado sensu stricto. A área possui 25 ha, sendo estes distribuídos entre as áreas de vegetação nativa de cerrado, reserva legal, área construída e buritizal.

$\mathrm{Na}$ área de estudo foram instaladas cinco transectos de $100 \mathrm{~m}$ cada, com interdistanciamento de $50 \mathrm{~m}$. Em cada uma das transecções foram coletadas quatro amostras de solos, com o auxilio de um trado de copo, em uma única profundidade, de $0-20 \mathrm{~cm}$, sendo estas encaminhadas ao Laboratório de Análise de Solos/LASO, do Centro de Ciências Agrárias, da Universidade Federal do Piauí/UFPI, para análise física e química, seguindo o Manual de Métodos de Análise de Solo (EMBRAPA, 1997).

As médias das características químicas e de textura das cinco transeções foram comparadas pelo método estatístico ANOVA (Análise de Variância) do programa SAS 2011, sendo as variâncias desiguais comparadas pelo teste de Tukey com nível de significância de 5\%.

\section{RESULTADOS E DISCUSSÃO}

A análise de solo nos transectos (Tabela 1.) estabelecidos revelou que as variáveis analisadas na área de estudo entre os transectos, permitiram considerar o solo com acidez elevada, com $\mathrm{pH}$ em $\mathrm{H}_{2} \mathrm{O}$ variando entre 4,63 a 4,82.

TABELA 1.Variáveis ambientais encontradas para os cinco transectos amostrados no Cerrado Sensu stricto sobre Neossolo Quartzarênico no Município de Caxia/MA.

\begin{tabular}{|c|c|c|c|c|c|c|c|}
\hline \multirow{2}{*}{ Variáveis } & \multicolumn{5}{|c|}{ Transectos } & \multirow[b]{2}{*}{ DP } & \multirow[b]{2}{*}{ CV (\%) } \\
\hline & 1 & 2 & 3 & 4 & 5 & & \\
\hline $\mathrm{pH}\left(\mathrm{H}_{2} \mathrm{O}\right)$ & 4,70 & 4,75 & 4,63 & 4,75 & 4,82 & 0,14 & 2,86 \\
\hline $\mathrm{Ca}^{2+}\left(\mathrm{cmo}_{d} / \mathrm{dm}^{3}\right)$ & 0,35 & 0,40 & 0,22 & 0,22 & 0,30 & 0.09 & 25,09 \\
\hline $\mathrm{Mg}^{2+}\left(\mathrm{cmo}_{\mathrm{d}} / \mathrm{dm}^{3}\right)$ & 0,40 & 0,28 & 0,32 & 0,25 & 0,42 & 0,18 & 56,24 \\
\hline $\mathrm{K}^{+}\left(\mathrm{cmo}_{d} / \mathrm{dm}^{3}\right)$ & 0,02 & 0,02 & 0,02 & 0,03 & 0,02 & 0,01 & 37,95 \\
\hline $\mathrm{Na}^{+}\left(\mathrm{cmo}_{d} / \mathrm{dm}^{3}\right)$ & 0,01 & 0,02 & 0,02 & 0,03 & 0,02 & 0,01 & 58,44 \\
\hline (valor S) $\mathrm{cmo}_{\mathrm{d}} / \mathrm{dm}^{3}$ & 0,75 & 0,70 & 0,63 & 0,50 & 0,77 & 0,20 & 28,45 \\
\hline $\mathrm{Al}^{3+}\left(\mathrm{cmo}_{d} / \mathrm{dm}^{3}\right)$ & 0,47 & 0,52 & 0,48 & 0,38 & 0,42 & 0,13 & 29,89 \\
\hline $\mathrm{H}^{+}\left(\mathrm{cmo}_{d} / \mathrm{dm}^{3}\right)$ & 3,47 & 4,30 & 3,85 & 2,90 & 4,00 & 0,67 & 13,83 \\
\hline $\mathrm{T}\left(\mathrm{cmo}_{\mathrm{c}} / \mathrm{dm}^{3}\right)$ & 4,70 & 5,52 & 4,95 & 3,77 & 5,20 & 0,85 & 13,80 \\
\hline $\mathrm{V} \%$ & 15,75 & 13,00 & 12,75 & 13,00 & 14,75 & 3,45 & 26,25 \\
\hline $\mathrm{S}+\left.\mathrm{Al}\right|^{3+}$ & 39,25 & 42,75 & 44,00 & 43,75 & 35,25 & 9,69 & 24,88 \\
\hline $\mathrm{P}^{+}\left(\mathrm{mg} \cdot \mathrm{L}^{-1}\right)$ & 6,50 & 8,50 & 4,00 & 2,25 & 2,25 & 3,01 & 39,42 \\
\hline $\mathrm{MO} \mathrm{g} / \mathrm{Kg}$ & 9,33 & 10,48 & 15,62 & 23,03 & 17,40 & 8,30 & 48,65 \\
\hline Areia Gro & 532,50 & 395,00 & 357,50 & 362,50 & 362,50 & 81,60 & 12,49 \\
\hline Areia Fi & 295,00 & 502,50 & 555,00 & 547,50 & 565,00 & 129,25 & 17,55 \\
\hline Silte $(\mathrm{g} / \mathrm{Kg})$ & 117,50 & 50,00 & 97,50 & 55,00 & 42,50 & 81,03 & 116,67 \\
\hline Argila $(\mathrm{g} / \mathrm{Kg})$ & 55,00 & 52,50 & 35,00 & 35,00 & 30,00 & 14,24 & 26,21 \\
\hline
\end{tabular}

Onde: $\mathrm{T}$ (capacidade de troca catiônica), $\mathrm{V}$ (saturação de bases), MO (matéria orgânica), DP (desvio-padrão), CV (coeficiente de variação).

Este solo de acordo com EMBRAPA (2006) tem elevada acidez por apresentar valores inferiores a 5 . Observou-se pouca variação entre os transectos, apresentando coeficiente de variação (CV) de 2,86\%. De acordo com EITEN (1972) na maioria das vezes os solos do Cerrado, são muito ácidos, com valores de $\mathrm{pH}$ 
entre 4,5 e 5,5. Os valores encontrados estão dentro da amplitude de 4,3 a 6,2 apregoada por LOPES (1984). Já os teores de Cálcio e Magnésio, permitiram classificá-los como baixos, variando de 0,22 a $0,40 \mathrm{cmo}_{\mathrm{C}} / \mathrm{dm}^{3}$ no Cálcio, enquanto que o Magnésio variou de 0,25 a $0,42 \mathrm{cmo}_{\mathrm{c}} / \mathrm{dm}^{3}$.

Quando se compara o cerrado sensu stricto de Caxias/Maranhão com as variáveis de solos analisadas por LINDOSO (2008), sob o mesmo tipo fisionômico de cerrado e de solo, tem-se que os valores de Cálcio e Magnésio são considerados baixos, entretanto os solos são de distinta acidez, sendo estes respectivamente de acidez elevada $(<5)$ e acidez média (5 a 5,9).

Os valores obtidos na matéria orgânica do solo (Tabela 1), variaram de 9,33 a 23,03 $\mathrm{g} / \mathrm{Kg}$ sendo considerados altos (EMBRAPA, 2006). Estes altos valores são justificados pelo acúmulo natural e incorporação do folhedo, pois a área sob estudo não apresenta histórico recente de queimadas na área pastejada. SILVA \& RESCK (1997) comentam que a Matéria Orgânica do solo, desempenha, importante papel na reciclagem de nutrientes, no tamponamento do solo contra alterações bruscas de $\mathrm{pH}$, na construção, na manutenção da estrutura e na adsorção e armazenamento de água. Dada a sua importância, deve ser conservada, manejada em um nível de equilíbrio que permita uma agricultura sustentável, pelo uso de métodos adequados de manejo de solo, compreendendo sistemas de preparo, rotação de culturas, adubação, estabelecimento de sistemas integrados de lavoura-pastagem, dentre outros.

Os valores da composição granulométrica foram maiores na composição textural da areia fina, seguida de areia grossa, silte e argila (Tabela 1). Os valores obtidos de areia foram superiores e os de silte e argila inferiores aos de LOSS (2011), onde este autor encontrou uma composição textural na profundidade de 0,0$20 \mathrm{~cm}$ da areia variando entre 240 a $260 \mathrm{~g} / \mathrm{Kg}^{-1}$, silte de 110 a $170 \mathrm{~g} / \mathrm{Kg}^{-1}$ e argila de 610 a $630 \mathrm{~g} / \mathrm{Kg}^{-1}$ em solos de cerrado sob diferentes sistemas de uso no município de Montividiu/GO. Já o teor de alumínio variou entre 0,38 a $0,52 \mathrm{cmo}_{\mathrm{c}} / \mathrm{dm}^{3}$, onde o Transecto (T2) apresentou o maior valor. Os valores de Fósforo variaram entre 2,25 a $8,79 \mathrm{mg} \cdot \mathrm{L}^{-1}$, valores estes considerados médios, enquanto no Potássio os valores foram baixos.

A toxidez de alumínio e os baixos teores de cálcio e de magnésio são características da maioria dos solos sob vegetação de Cerrado. Como consequência, as plantas são pouco desenvolvidas, com sistema radicular raquítico, o que limita o aproveitamento da água e dos fertilizantes adicionados ao solo. A correção da acidez se faz necessária para se obter melhor produtividade das culturas e menores perdas de adubos (SOUSA et al. 1985).

\section{CONCLUSÃO}

Foi observado que Neossolos Quartzarênicos do Município de Caxias/MA apresentaram teores de Matéria Orgânica do Solo típicos aos desta classe de solos, alta acidez, baixo teor de nutriente e textura arenosa. Pode se inferir que esses maiores valores tenham ocorrido pela deposição de folhedo e não ocorrência de queimadas recentes na área. O solo estudado por suas características deve ser utilizado para conservação da flora e fauna residente. 


\section{REFERÊNCIAS}

ADÁMOLI, J.; MACEDO, J.; AZEVEDO, L. G. de, NETO, J. M. Caracterização da região dos cerrados. In: GOEDERT. W. J. (Ed.). Solos dos cerrados: tecnologias e estratégias de manejo. São Paulo: Nobel,1985. p. 33-74.

ASSUNÇÃO, S. L.; FELFILI, J. M. Fitossciologia de um fragmento de cerrado sensu stricto na APA do Paranoá, DF, Brasil. Acta Botânica Brasilica, Porto Alegre, v. 18, n. 4, p. 903-909, 2004.

BERTONI, J.; LOMBARDI NETO, F. Conservação do solo. Piracicaba: Livroceres, 1985. $392 \mathrm{p}$.

BRADY, N. C. Natureza e propriedades dos solos "The nature and proprieties of soils". 7.ed. Tradução Antonio B. Neiva Figueiredo. Rio de Janeiro: Freitas Bastos, 1989. $890 \mathrm{p}$.

BRASIL. Ministerio do Meio Ambiente. Relatório técnico de monitoramento do desmatemento do bioma cerrado, 2002 a 2008: Dados Revisados. 2009. Disponível:<http://siscom.ibama.gov.br/monitorabiomas/cerrado/Relatorio\%20tecnico Monitoramento\%20Desmate_Bioma\%20Cerrado_CSR_REV.pdf>. Acesso em: 19 abr. 2011.

CONCEIÇÃO, G. M.; SILVA, E. R.; SILVA, M. C. C.; SILVA, R. P.; CASTRO, L. M. R. determinação de macronutrientes $(\mathrm{N}, \mathrm{P}, \mathrm{K}, \mathrm{CA}, \mathrm{S}, \mathrm{MG})$ nas espécies de poaceae de uma área de Cerrado Maranhense. ENCICLOPÉDIA BIOSFERA, Centro Científico Conhecer - Goiânia, v.9, n.17; p. 2013.

COUTINHO, L. M. O bioma cerrado. In: KLEIN, A. L. (ed.) Eugen Warming e o cerrado brasileiro: um século depois. Editora UNESP, São Paulo, p. 77-90, 2002.

EITEN, G. The cerrado vegetation of Brasil. The Botanical Review, v. 38, n. 2, p. 201-341, 1972.

EMBRAPA - Empresa Brasileira de Pesquisa Agropecuária. Sistema Brasileiro de Classificação de Solos. $2^{\underline{a}}$ ed. Brasilia: Embrapa Produção de Informação, Rio de Janeiro, Embrapa Solos, 312 p. 2006.

EMBRAPA/CENTRO NACIONAL DE PESQUISA DO SOLO. Manual de métodos de análises de solo. 2.ed. Brasília: Embrapa Produção de Informação, Rio de Janeiro, Embrapa Solos, 1997. 212 p.

LINDOSO, G. S. Cerrado sensu stricto sobre neossolo quartzarênico: fitogeografia e conservação. 2008. 170 f. Tese (Doutorado) - Universidade de Brasília, Brasília, 2010.

LOPES, A. S. Solos sob “cerrado". Piracicaba: POTAFOS, 1984. 162 p.

LOSS, A. Dinâmica da matéria orgânica, fertilidade e agregação do solo em áreas sob diferentes sistemas de uso no cerrado Goiano. 2011. $122 \mathrm{f}$. Tese (Doutorado) - Universidade Federal Rural do Rio de Janeiro, Instituto de Agronomia, Seropédica, 2011. 
NETTESHEIM, F. C.; CARVALHO, D. C.; FONSECA, C. C.; NUNES, R. S.; CAVALCANTI, D. M.; GABRIEL, M. M.; MENEZES, L. F. T. Estrutura e florística do estrato arbóreo no cerrado sensu stricto de Buritis, Minas Gerais, Brasil. Rodriguésia, Rio de Janeiro, v. 61, n. 4, p. 731-747, 2010.

PINTO M. N.; BARROS, J. G. C. Conservação das águas e solos. In: DIAS, B.F. S. (Coord.). Alternativas de desenvolvimento dos Cerrados: manejos e Conservação dos recursos naturais renováveis. Brasília: Fundação Pró-Natura, 1996. p. 63-65.

REATTO, A.; MARTINS, E. S. Classes de solo em relação aos controles da paisagem do bioma cerrado. In: SCARIOT, A.; SOUSA-SILVA, J. C.; FELFILI, J. M. (Org.). Cerrado: ecologia, biodiversidade e conservação. Brasília: Ministério do Meio Ambiente, 2005. p. 47-60.

RIBEIRO, J. F.; WALTER, B. M. T. As principais fitofisionomias do Bioma Cerrado. In: SANO, S. M.; ALMEIDA, S. P.; RIBEIRO, J.F. (Ed.). Cerrado: ecologia e flora. Planaltina: Embrapa Cerrados, 2008. p. 151-212.

RIBEIRO, J. F.; WALTER, B. M. T. Fitofisionomias do bioma Cerrado. In: SANO, S. M.; ALMEIDA, S. P. (Ed.). Cerrado: ambiente e flora. Planaltina: EMBRAPA/CPC, 1998. p. 29-47.

SILVA, J. E. \& RESCK, D. V. S. Materia orgânica do solo, p. 467-523. In: Biologia dos solos do cerrado. VARGAS, M. A. T. \& HUNGRIA, M. Planaltina: EMBRAPA/CPAC, 1997, 524 p.

SOUZA, D. M. G.; CARVALHO, L. J. C. B. Nutrição mineral de plantas. In: GOEDERT, W. J. (Eds.). Solos dos cerrados: tecnologias e estratégias de manejo. Planaltina: EMBRAPA/Cerrados,1985. p.75-98. 